\title{
Modulation of brain activity during phonological familiarization
}

\author{
Majerus Steve(1)(2), Van der Linden Martial ${ }^{(1)(3)}$,Collette Fabienne( ${ }^{(1)(2)}$,Laureys Steve ${ }^{(2)(4)}$, \\ Poncelet Martine ${ }^{(1)}$, Degueldre Christian ${ }^{(4)}$,Delfiore Guy ${ }^{(4)}$,Luxen André(4),Salmon Eric ${ }^{(4)}$
}

(1) Department of Cognitive Sciences, University of Liège, Belgium

(2) Fonds National de la Recherche Scientifique, Belgium

(3) Cognitive Psychopathology Unit, University of Geneva, Switzerland

(4) Cyclotron Research Unit, University of Liège, Belgium

\begin{abstract}
We measured brain activity in 12 adults for the repetition of auditorily presented words and nonwords, before and after repeated exposure to their phonological form. The nonword phoneme combinations were either of high (HF) or low (LF) phonotactic frequency. After familiarization, we observed, for both word and nonword conditions, decreased activation in the left posterior superior temporal gyrus, in the bilateral temporal pole and middle temporal gyri. At the same time, interaction analysis showed that the magnitude of decrease of activity in bilateral posterior temporal lobe was significantly smaller for LF nonwords, relative to words and HF nonwords. Decrease of activity in this area also correlated with the size of behavioral familiarization effects for LF non-words. The results show that the posterior superior temporal gyrus plays a fundamental role during phonological learning. Its relationship to sublexical and lexical phonological processing as well as to phonological short-term memory is discussed.
\end{abstract}

Keywords: Phonological processing; Phonological learning; Temporal lobe; Superior temporal gyrus; PET

\section{Introduction}

In the past 15 years, many functional neuro-imaging studies have explored the neural substrates of phonological and lexico-semantic representations in the adult healthy brain. Brain regions underlying the sublexical phonological processing of speech input have been shown to be located primarily in the left or bilateral superior temporal area, and more precisely in the superior temporal sulcus and the posterior superior temporal gyrus; these regions are activated when participants listen to both meaningful and meaningless speech (e.g., nonwords), but not when listening to acoustically matched non-verbal sounds (e.g., Binder et al., 2000; Binder \& Price, 2001; Burton, Small, \& Blumstein, 2000; Demonet et al., 1992; Demonet, Price, Wise, \& Frackowiak, 1994; Hickok et al., 2000; Jacquemot, Pal-lier, Le Bihan, Dehaene, \& Dupoux, 2003; Ja“ncke, Wu“stenberg, Scheich, \& Heinze, 2002; Majerus et al., 2002; Mazoyer et al., 1993; Mummery, Ashburner, Scott, \& Wise, 1999; Specht et al., 2003). The left posterior superior temporal sulcus also responds to spectrally distorted speech that is completely unintelligible, but retains some phonetic information (Scott, Catrin Blank, Rosen, \& Wise, 2000). Although there is still some ongoing controversy about the question of whether these regions really reflect processing at the phonological level or whether they are related to the processing of those acoustic features that are specific to human speech sounds (e.g., the rapid temporal characteristics of stop consonants, see for example Joanisse \& Gati, 2003), most authors agree that the posterior superior temporal lobe underlies the processing of the sound structure of human speech. On the other hand, lexico-semantic processing of speech stimuli involves activation of mainly left-sided middle and inferior temporal cortex (BA 21 and BA 20/37), inferior parietal cortex (BA 39, angular gyrus), as well as inferior frontal and dorsal prefrontal regions (BA 46/47 and BA 8/9/10) (Binder et al., 1999; Binder, Frost, Hammeke, Rao, \& Cox, 1996; Demonet et al., 1992, 1994; Howard et al., 1992; Majerus et al., 2002; Perani et al., 1996; Price et al., 1996; Scott et al., 2000). These regions are activated in different tasks that implicate access to the lexico-semantic content of verbal stimuli, such as passive listening to intelligible and meaningful words or sentences, as well as object naming and 
semantic judgment tasks.

Relative to the abundance of PET and fMRI studies on phonological and lexico-semantic

representations, it is somewhat surprising that very few of these studies have explored the neural substrates underlying the acquisition of these representations (see also Breiten-stein \& Knecht, 2002). This is however a very important point, as understanding the brain mechanisms that underlie the construction of language representations is likely to influence the way we interpret how the different brain regions process these language representations, once they are installed.

Some indirect and controversial data for neural substrates underlying language learning can be found in the neuro-imaging literature of aphasic patients who underwent language therapy (e.g., Leger et al., 2002; Musso et al., 2000; Thompson, 2000). These studies explored change of brain activity in different language tasks as a result of a more or less specific speech therapy program. They showed either a brain activation pattern that returned back to relatively normal left hemisphere activation surrounding the lesioned area (for a naming task; Leger et al., 2002), or spatially extended activity in the left hemisphere inferior temporal regions (for sentence processing tasks; Thompson, 2000) or activation in the homotopic right superior posterior temporal regions (for sentence processing tasks; Musso et al., 2000; Thompson, 2000). The neuro-imaging literature on language learning in aphasic patients is however problematic for two reasons: (1) the brain activations reported in these studies are not directly associated with language learning itself, but reflect the distance effects of rather global speech therapy programs on naming or sentence comprehension performance; (2) language learning mechanisms that happen in a left-hemisphere damaged brain are not necessarily representative of brain mechanisms underlying normal language learning, but might reflect compensatory mechanisms occurring only in the pathological brain. Similar problems are associated with developmental neuroimaging studies that studied the brain substrates of language processing in children of different ages. These studies generally show that the temporal, parietal, and frontal regions underlying phonological and semantic processing tasks in adults are already activated in infants, although these activations seem to be larger and less lateralized to the left hemisphere than will be the case in older children and adults (e.g., Booth et al., 2001; Dehaene-Lambertz, Dehaene, \& Hertz-Pannier, 2002; Holland et al., 2001). However, these developmental neuro-imaging studies still do not directly explore the learning mechanisms that happen during language development.

A recent fMRI study investigated verbal learning in healthy adults. Clark and Wagner (2003) related brain activation for making syllable decisions on visually presented words and nonwords to subsequent memory scores for the same words and nonwords. They observed that activation in superior and inferior parietal cortex as well as in left inferior prefrontal cortex positively correlated with subsequent memory scores. Furthermore, this effect was greater in the left inferior prefrontal cortex for nonwords compared to words. As the parietal and left inferior prefrontal cortex are respectively associated with phonological working memory storage and control processes (Awh et al., 1996; Davachi, Maril, \& Wagner, 2001; Jonides et al., 1998; Paulesu, Frith, \& Frackowiak, 1993; Smith \& Jonides, 1998), this study suggests that the phonological control component of working memory plays a central role in the encoding of novel phonological representations in the language network, as has also been shown by studies on aphasic patients (Corne-lissen et al., 2003). However, this study still sheds no light on what is directly happening in superior, middle, and inferior temporal language areas during the learning of new language representations. Furthermore, a syllable counting task on visually presented words and non-words cannot be considered as a very naturalistic language learning task.

As a whole, the present literature is relatively poor with regard to the brain mechanisms that underlie the acquisition of phonological and semantic language representations. The aim of the present study was to explore the neural substrates of a very basic language learning mechanism: adaptation of brain activation to the repeated exposure to familiar (words) or unfamiliar (nonwords) phonological information. The learning mechanism that comes closest to the paradigm used in this study is repetition priming, which has been more extensively explored in functional neuro-imaging studies on memory. Repetition priming is characterized by faster and more accurate processing of stimuli following repetition. Repetition priming for familiar information has been associated with decreased brain response and has been interpreted to reflect more efficient, focused, and faster activation of neural representations (see Hen-son \& Rugg, 2003, for a critical review). For example, repeated semantic judgment for the same auditorily presented object name results in decreased activity in the bilateral superior temporal area, reflecting repetition priming at the phonological level, and in the left temporal, left inferior prefrontal and medial prefrontal cortex reflecting repetition priming at the semantic level (Buck-ner, Koutstaal, Schacter, \& Rosen, 2000; Marinkovic et al., 2003). One study explored repetition priming at the phonological and lexico-semantic level for both familiar and unfamiliar verbal information. Haist et al. (2001) compared repetition priming for visually presented words, pseudohomophones (nonwords sounding like a familiar word, e.g., jale) and pseudowords (e.g., muvel) 
in a lexical decision task. The authors hypothesized that lexical decision on visually presented words, pseudoho-mophones and pseudowords would also engage phonological processes in the superior temporal lobe. They indeed observed decreased activation in the left primary auditory cortex for the repeated presentation of words and pseudohomophones (which sound like words), but not for pseudowords. Although the precise meaning of these activations is not quite clear, these results suggest at least that repetition priming may have different effects on brain activation depending on the lexical status of the verbal information that is presented. However, repetition priming for auditorily presented familiar and unfamiliar verbal information (such as words and nonwords) has not yet been directly explored.

In the present PET study, we compared brain activation for the repetition of auditorily presented words and nonwords, before and immediately after a familiarization phase that took place between two scanning blocks. During the familiarization phase, the participants were repeatedly presented 20 word or nonword stimuli and they were required to repeat each stimulus as accurately and as fast as possible after each presentation. In the subsequent scanning block, the same 20 stimuli were represented and the participants were once again required to repeat them as fast and as accurately as possible. We were interested in familiarization-related changes in brain activity in phonological and lexico-se-mantic language processing areas for the word and non-word stimuli. For words, we expected a reduction in brain activity in phonological processing areas, i.e., the posterior superior temporal gyrus and posterior superior temporal sulcus, as well as in lexico-semantic processing areas, i.e., anterior superior temporal, middle, and inferior temporal, parietal (angular gyrus), and prefrontal cortices. For nonwords, which by definition are meaningless, we expected that familiarization should induce brain activity changes mainly in the superior temporal phonological processing areas, but less so in lexico-semantic processing areas. Furthermore, we hypothesized that these changes in brain activity could be less pronounced for nonwords than for words, according to the study by Haist et al. (2001). Finally, we contrasted nonwords containing either frequent or infrequent phoneme associations relative to the phonotactic structure of French phonology. Thus the nonwords used in this study varied with respect to initial phonological familiarity. In previous work, we had shown that the right posterior superior temporal gyrus responded differentially to phonotactically frequent (HF) and infrequent (LF) nonwords during a passive listening and a repetition task, and that response latencies for repeating HF nonwords were shorter than for LF nonwords (Majerus et al., 2002). If phonological familiarization affects words and nonwords differentially, then this difference should be most pronounced between words and phono-tactically infrequent nonwords (LF), with an intermediate effect for phonotactically frequent nonwords (HF). The need to obtain response latency measures via a voice-key within the scanner determined our choice of PET imaging rather than fMRI.

\section{Methods}

\subsection{Subjects}

Twelve French-speaking right-handed male volunteers (age 18-25 years) gave their written informed consent to take part in this study, which was approved by the Ethics Committee of the Faculty of Medicine of the University of Lie'ge. None had a significant medical history nor used any centrally acting medication.

\subsection{Cognitive tasks}

Three lists of bisyllabic stimuli were created: 80 low phonotactic frequency (LF) nonwords, 80 high phono-tactic frequency (HF) nonwords, and 80 words. Each stimulus had the same syllabic structure CVCCVC. The LF nonwords were constructed using CV and VC diphones that are quite rare in French (e.g., in the non-word /fœglo $\left[/\right.$, the diphones /fœ/, /œg/, /lo/, and /o $\int /$ are not very frequent in French). On the other hand, the HF nonwords contained CV and VC diphones which are quite frequent (e.g., in the nonword /pebmyn/, the diphones /pe/, /eb/, /my/, and /yn/ are very frequent in French). Furthermore, the diphones of the HF non-words had the same phonotactic frequency as the di-phones of the words ${ }^{1}$ used in this study. The diphone frequencies were taken from a phonetic database of French by Tubach and Boe (1990, Un corpus de transcription phonetique). This database was developed on the basis of a phonetic transcription of formal and informal conversations between French-speaking subjects. To measure phonotactic frequency, we used the simple diphone frequency counts listed in the database. There was a significant difference in summed diphone frequency counts 
for the CV and VC diphones in the first syllable of the HF versus LF nonwords (2106 vs. 138; $z=$ $14.57, p<.0001)$, and in the second syllable (2293 vs. $154 ; z=17.96, p<.0001)$; there was no difference in summed diphone frequency counts for the first syllable of HF nonwords versus words ( 2106 vs. $2255 ; z=1.09$, n.s.) and for the second syllable ( 2293 vs. $2345 ; z=.38$, n.s.). Furthermore, the consonants and vowels used for the three lists were sampled from the same pool of phonemes. The stimuli were recorded by a trained native French female speaker. All stimuli were spoken in isolation. The stimuli were low-pass filtered at $4.8 \mathrm{kHz}$ and digitized at a sampling rate of $11,025 \mathrm{~Hz}$. All stimuli were edited into individual files and stored on computer disk. There were no significant differences in mean stimulus duration for the different stimulus lists, $F(2,158)<1$, n.s. (words: 1059ms; HF nonwords: 1066ms; and LF nonwords: 1079 ms).

Stimulus presentation was controlled via E-Prime beta software (Psychology Software Tools, 2003) installed on a PC desktop computer. Stimuli were presented via headphones. Each subject was administered four blocks of 20 words, four blocks of $20 \mathrm{HF}$ non-words, and four blocks of $20 \mathrm{LF}$ nonwords. The subjects were told that they had to repeat as accurately and as quickly as possible the words or nonwords they were going to hear. Behavioral data were collected by recording the subjects' responses via a digital-to-analog tape recorder connected to a microphone; response latencies (time between the onset of the stimulus and the onset of the subjects' responses) were also recorded via a second microphone connected to E-Prime beta software. In each block, the stimuli were presented at a rate of one item every $4 \mathrm{~s}$. The order of presentation of the blocks and of the stimuli within each block was randomized. Before each block, the subjects were told by the experimenter whether they would be presented words or nonwords. They were explicitly informed about the lexical status of the stimuli they would hear in order to avoid lexical search processes when presenting nonwords while they might have been expecting to hear words.

\subsubsection{Familiarization}

After the first six scanning blocks (comprising two word blocks, two HF nonword blocks, and two LF non-word blocks), the participants were familiarized to the stimuli presented during the next scanning block. The familiarization phase consisted in six presentations of the 20 stimuli for the next scanning block, and the participants were asked to repeat the stimuli after each presentation. The order of presentation of the stimuli during the familiarization phase was randomized and the rate of presentation was one item every $4 \mathrm{~s}$. Response accuracy and latency times were recorded using the same procedure as during the scanning blocks. After six repetitions of the stimulus set (total duration: $8 \mathrm{~min}$ ), the scanning session began. The order of presentation of the blocks (words, HF nonwords, and LF nonwords) during the six familiarization scans was randomized. For each condition, the 80 stimuli were randomly assigned to two sets of $2 \times 20$ stimuli. For half of the participants, the first set was presented during the first six scan runs, and the second set was presented during the six familiarization scan runs. For the other half of the participants, the stimulus set was reversed.

To maximize the difference between novel and familiarization scans, we chose to present novel and familiarization scans in a successive order rather than a counterbalanced order. When presenting familiarization scans before novel scans, the phonological learning processes operating during the familiarization scans could also contaminate novel items as their phonological structure is very similar to the stimuli used during familiarization. Experimental studies have indeed shown that a continuous exposure to new phonological information will affect the later processing of nonwords, even if these nonwords were not explicitly presented during phonological exposure (Majerus, Van derLinden, Mulder, Meulemans, \& Peters, 2004; Saffran, Aslin, \& Newport, 1996a, 1996b, 1997). However, in order to avoid that the familiarization effect was confounded by time in the present experiment, time was introduced as a covariate in the subsequent analyses (see the Data analysis section for further details). Furthermore, in order to completely rule out the possibility that the familiarization effects we would observe are simply related to an habituation to the procedure, we had also reanalyzed the data from a previous PET study where participants had to repeat and listen to exactly the same word, HF nonword, and LF nonword stimuli as those used in the present study during 12 scans, but without any familiarization procedure (Majerus et al., 2002). We determined which brain regions showed increased or decreased brain activity as a function of scan number. This reanalysis revealed that the only region that showed time-related decrease of activity was located in the right medial temporal lobe area [coordinates: $34-3-23$ ]. Increase of activation as a function of time was observed in the right middle frontal gyrus [coordinates: 60 14 32]. Most notably, none of these regions concerned those brain areas of interest in the present study: the superior, middle, and inferior temporal lobes. 


\subsection{Positron emission tomography scanning}

PET data were acquired on a Siemens CTI 951 R 16/ 31 scanner in 3D mode. The subject's head was stabilized by a thermoplastic facemask secured to the head holder (Truscan imaging, MA), and a venous catheter was secured in a left antebrachial vein. First, a 20-min transmission scan was acquired for attenuation correction. Then, regional cerebral blood flow, taken as a marker of local neuronal activity (Jueptner \& Weiller, 1995), was estimated during 12 emission scans. Each scan consisted of two frames: a 30-s background frame and a 90-s acquisition frame. The slow intravenous water $\left(\mathrm{H}_{2}{ }^{15} \mathrm{O}\right)$ infusion began $10 \mathrm{~s}$ before starting the second frame. Six mCi $(222 \mathrm{MBq})$ in $5 \mathrm{~cm}$ saline were injected over a period of $20 \mathrm{~s}$ for each scan. The infusion was totally automated in order so as not to disturb the subject during the scanning period. Data were reconstructed using a Hanning filter (cut-off frequency: .5 cycle/pixel) and corrected for attenuation and background activity. Each experimental task (block) corresponded to one scan acquisition and began $10 \mathrm{~s}$ before starting the acquisition frame.

\subsection{Data analysis}

Data were analyzed and transformed using the statistical parametric mapping software (SPM99; Wellcome Department of Cognitive Neurology, Institute of Neurology, London, UK) implemented in MATLAB (Mathworks, Sherborn, MA). First, spatial transformations were performed to accommodate within-subject head-movement in scan replications and inter-subject differences in gyral and functional anatomy. For each subject, all scans were realigned together, and normalized to a standard PET template (Friston et al., 1995a). Spatial registration and normalization of images conform to the space defined by the ICBM, NIH P-20 project, and approximate that of the space described in the stereotaxic atlas of Talairach and Tournoux (1988). Finally, PET images were smoothed using a Gaussian kernel of $16 \mathrm{~mm}$ full width at half maximum.

We first looked for areas that responded differentially to the type of stimuli (words [W], high frequency non-words $[\mathrm{HF}]$, and low frequency nonwords $[\mathrm{LF}]$ ), prior to familiarization. These were identified by different subtraction analyses $([\mathrm{W}-\mathrm{HF}],[\mathrm{W}-\mathrm{LF}],[\mathrm{HF}-\mathrm{W}],[\mathrm{LF}-\mathrm{W}]$, and $[\mathrm{HF}-\mathrm{LF}])$. Then we determined brain regions consistently supporting the task effect (familiarization [fam]) for the different stimulus conditions separately also using a set of subtraction analyses ([W — famW], [HF famHF], [LF — famLF], [famW - W], [famHF - HF], and [famLF - LF]). Next, we conducted interaction analyses in order to determine brain regions that were differentially activated for HF and LF nonwords relative to words, before and after familiarization $\quad([$ famLF $-\mathrm{LF}]-[$ famW $-\mathrm{W}]$; $[\mathrm{famHF}-\mathrm{HF}]-[\mathrm{fam} W-\mathrm{W}] ;[\mathrm{famLF}-\mathrm{LF}]-[\mathrm{famHF}-\mathrm{HF}] ;[\mathrm{famHF}-\mathrm{HF}]-[\mathrm{famLF}-$ LF $]$ [ $\quad[$ famW $-\mathrm{W}]-[$ famHF $-\mathrm{HF}]$; and $[\mathrm{famW}-\mathrm{W}]-[$ famLF $-\mathrm{LF}])$. Finally, we estimated the correlation between brain activity and the reaction time data before and after familiarization for the different stimulus conditions, by correlating brain activity of the contrasts [famW - W], [famHF — HF], and [famLF — LF] with the reaction time difference between the respective conditions.

The condition and subject effects were estimated according to the general linear model at each voxel (Fris-ton et al., 1995b), using a mixed effect model (Holmes \& Friston, 1998). In SPM99, the mixed or random effect model is a two-step procedure based on the approach of mean summary statistics on repeated measures. It was applied to accommodate intra-individual then inter-individual variability of PET data, explicitly accounting for subject by condition interaction effects. At the first step, areas of significant change were determined at the within-subject level using linear contrasts of condition estimates. Adjustment for one subject was made independently from other subjects by using ANCOVA adjustment of the global activity. Scan number was introduced as a nuisance variable regressor; this was necessary as the first six scans were all baseline blocks, and the last six scans were all familiarization blocks. For each individual, subtraction and interaction contrasts were computed separately. The resulting estimates (i.e., individual contrast images) fitted the within-subject component of the variance. At the second step of the analysis, the residual between-subject variance was assessed. Individual contrast images were entered in the design matrix using a multiple regression model. For the correlation analysis, individual contrast images were entered in the design matrix using a simple regression model; the difference between reaction times corresponding to the two conditions represented by each individual contrast image was then introduced as a regressor.

The resulting set of voxel values for each contrast constituted a map of the T statistic [SPM(T)], threshol-ded at $p \leq .001$. If not otherwise specified, statistical inferences were obtained at the voxel level (in terms of peak height at $p<.05$ ), corrected for multiple comparisons. The significance was set 
at the level of $p<.05$, corrected for small search volume (10 mm radius spherical volume) on published coordinates for brain regions where we had a priori knowledge on their involvement in either phonological or lexico-semantic processing during repetition and passive listening tasks of word and nonword stimuli. For lexico-semantic processing, these were the left anterior superior temporal sulcus (BA 22/38), anterior middle (BA 21), and posterior inferior temporal gyri (BA 20/37), inferior parietal (BA 39, angular gyrus) (Binder et al., 1996, 1999, 2000; Howard et al., 1992; Majerus et al., 2002; Perani et al., 1996; Petersen, Fox, Posner, Mintun, \& Raichle, 1988, 1989;Price et al., 1996; Scott et al., 2000). For phonological processing, the coordinates of interest were situated in the posterior superior temporal gyrus bilaterally (BA 22 and BA 22/40) and the superior temporal sulcus bilaterally (BA 22), according to the coordinates published in Binder et al. (2000), Binder and Price (2001), Burton et al. (2000), Hickok et al. (2000), Jacquemot et al., 2003; Ja“ncke et al. (2002), Majerus et al. (2002), Mummery et al. (1999), Scott et al. (2000), and Specht et al. (2003).

\section{Results}

\subsection{Behavioral results}

Regarding response latencies, a 2 (before or after familiarization) x 3 (word, HF nonword, LF nonword) repeated measures ANOVA showed a main effect of familiarization, $\mathrm{F}(1,11)=43.84, \mathrm{p}<$ .001 , and a main effect of stimulus condition, $\mathrm{F}(2,22)=75.02, \mathrm{p}<.001$. Tu-key post-hoc comparisons showed that response latencies were significantly shorter after familiarization for all three stimulus conditions, words were processed faster than HF and LF nonwords, and HF nonwords were processed faster than LF nonwords, both before and after familiarization (see Table 1). Regarding response accuracy, a similar repeated measures ANOVA revealed no effect of familiarization, $\mathrm{F}(1,11)=2.84$, n.s., but an effect of stimulus condition, $F(2,22)=17.80, p<.001$. Post hoc comparisons showed that there was a slight advantage for words over LF nonwords before and after familiarization, and for words over HF nonwords after familiarization (see Table 1). To summarize, we observed a robust effect of both familiarization and stimulus type on response latencies. Response accuracy overall was very high and was not significantly influenced by familiarization; only a modest effect of stimulus condition was observed.

\subsection{Imaging results}

3.2.1. Subtraction analyses: Differences in brain activation between words, HF nonwords, and LF nonwords prior to familiarization

Words activated more than HF and LF nonwords an area in the left inferior temporal lobe. HF nonwords activated more than words the lateral surface of the left superior temporal lobe. No other differences were observed between the three types of stimuli prior to familiarization (see Table 2). 3.2.2. Subtraction analyses: Familiarization-modulated brain activity for words Regions that showed diminished activation after familiarization were located in the bilateral superior temporal lobe (see Table 3 and Fig. 1). They included the left posterior superior temporal gyrus and the bilateral superior temporal sulcus.

Diminished activation was also observed in regions previously associated with lexico-semantic processing: the bilateral temporal pole, the bilateral middle temporal gyrus, and left inferior temporal gyrus. Significantly increased activation after familiarization was observed in the right precuneus (Table 4); as shown in Fig. 1, at uncorrected $p$ levels, we also observed activation in the prefrontal cortex (left superior frontal [ - 22 58 -3], right middle frontal [34 14 45], and right precentral [28 - 1 53]), the parietal cortex (left inferior parietal [ $-40-4445]$ ), and the occipital cortex (right inferior occipital $[30-61-7])$.

\subsubsection{Subtraction analyses: Familiarization-modulated brain activity for HF nonwords}

As for words, familiarization with HF nonword stimuli induced diminished activity in the left posterior superior temporal gyrus, the bilateral superior temporal sulcus, the bilateral temporal pole, as well as the middle temporal gyrus (see Table 3 ). Significantly increased activity was observed in extratemporal brain regions, mainly the right cingulate (see Table 4); as for words, increase of activity was 
also observed in prefrontal cortex (bilateral superior frontal [42 51 7; - $1660-3]$, right precentral [46 -11 52]), the parietal cortex (left inferior parietal [ $-40-4445]$ ) and the occipital cortex (right inferior occipital [44 -58 36]) at uncorrected p-levels (Fig. 1).

No increased activation was observed in the superior or middle temporal areas, even when lowering the statistical threshold $(p<.001$, uncorrected).

Table 1 Response latencies and accuracy, as a function of stimulus conditionand familiarization (mean and standard deviations)

\begin{tabular}{llll}
\hline Response latency $(\mathrm{ms})$ & Word & HF nonword & LF nonword \\
Before familiarization & & & \\
After familiarization & $1352(114)$ & $1513(149)$ & $1593(160)$ \\
Accuracy (proportion) & $908(211)$ & $1108(294)$ & $1216(241)$ \\
Before familiarization & & & \\
After familiarization & $.98(.02)$ & $.94(.05)$ & $.91(.07)$ \\
& $.99(.01)$ & $.95(.05)$ & $.93(.03)$
\end{tabular}

Table 2 Subtraction analyses: Differences in brain activation between words, HF nonwords, and LF nonwords, prior to familiarization

Brain area

Words - HF nonwords

L Inferior temporal (BA 20)

Words - LF nonwords

L Inferior temporal (BA 20)

HF nonwords - Words

L Posterior superior temporal (BA 22)

LF nonwords - Words

No suprathreshold activation

HF nonwords - LF nonwords

No suprathreshold activation

${ }^{*} p .05$, small volume corrections.
Stereotaxic coordinates

Z-value

$-46$

$\mathrm{y}$

$\begin{array}{llll}-38 & -24 & -19 & 3.58 *\end{array}$

$\begin{array}{llll}-67 & -23 & 12 & 3.29 *\end{array}$
$4.11^{*}$ 
Table 3 Subtraction analyses: Brain regions less activated after familiarization for words, HF nonwords, and LF nonwords

Brain area

$\begin{array}{lll}\text { Stereotaxic coordinates } & & \text { Z-value } \\ \mathrm{x} & \mathrm{y} & \mathrm{z}\end{array}$

Words

L Posterior superior temporal (BA 22/40)

L Posterior STS (BA 22)

L Anterior temporal (BA 38)

L Middle temporal (BA 21)

L Inferior temporal (BA 20)

R Posterior STS (BA 22)

R Anterior temporal (BA 38)

R Middle temporal (BA 21/38)

$-67$

HF nonwords

L Posterior superior temporal (BA 22)

L Posterior STS (BA 22)

L Middle temporal (BA 21/38)

R Posterior STS (BA 22)

$\begin{array}{llll}-67 & -40 & 20 & 5.36 \\ -61 & -43 & 2 & 4.47^{*} \\ -48 & 28 & -26 & 5.12 \\ -55 & -7 & -18 & 5.51 \\ -44 & -20 & -19 & 4.75 \\ 69 & -19 & 1 & 3.46_{-}^{*} \\ 46 & 22 & -31 & 4.78^{-} \\ 56 & 10 & -26 & 5.52\end{array}$

R Anterior temporal (BA 38)

R Middle temporal (BA 21)

LF nonwords

L Posterior superior temporal (BA 22)

L Middle temporal (BA 21)

R Anterior temporal (BA 38)

R. Middle temporal (BA 21)

$\begin{array}{llll}-69 & -42 & 19 & 4.88 \\ -69 & -33 & 7 & 4.36^{*} \\ -63 & -43 & 2 & 4.33^{*} \\ -55 & 5 & -15 & 3.89^{*} \\ 61 & -16 & -4 & 3.79^{*} \\ 51 & 11 & -21 & 4.96 \\ 44 & 3 & -27 & 5.42 \\ & & & \\ -69 & -38 & 22 & 4.30_{-}^{*} \\ -51 & -3 & -17 & 3.68_{-}^{*} \\ 46 & 22 & -28 & 4.90^{*} \\ 46 & 2 & -29 & 4.11_{-}\end{array}$

If not otherwise specified, results are significant at $\mathrm{p}<.05$, corrected for multiple comparisons. STS, superior temporal sulcus. * $\mathrm{p}<.05$, small volume corrections.

Table 4 Subtraction analyses: Brain regions more activated after familiarization for words, $H F$ nonwords and LF nonwords

Brain area

Words

R Precuneus (BA 7)

R Precuneus (BA 19)

R Precuneus (BA 31)

HF nonwords

R Cingulate (BA 23/31)

LF nonwords

R Precuneus (BA 31)

Results are significant at $p<.05$, corrected for multiple comparisons.

$\begin{array}{lccc}\begin{array}{l}\text { Stereotaxic coordinates } \\ \mathrm{x}\end{array} & \mathrm{y} & \mathrm{Z} \\ & & & \\ 14 & -59 & 32 & 5.20 \\ 30 & -74 & 42 & 4.78 \\ 10 & -65 & 25 & 4.90 \\ 12 & -43 & 26 & 4.80 \\ 14 & & & \\ 14 & -59 & 32 & 5.69\end{array}$

3.2.4. Subtraction analyses: Familiarization-modulated brain activity for LF nonwords

After familiarization with LF nonwords, reduced activity was observed in the left posterior superior temporal gyrus, in the right temporal pole as well as in the bilateral middle temporal gyrus (see Table 3). Contrary to words and HF nonwords, no diminished activation was observed in right superior temporal sulcus.

Increased activation after familiarization was observed exclusively in extra-temporal brain regions, mainly the right precuneus (see Table 4); as for the other two stimulus types, increased activation was also observed at uncorrected $p$ levels in the prefrontal cortex (left superior frontal [ $-2452-1$ ], right precentral [57 - 12 37]), the parietal cortex (left inferior parietal [ - 40 - 3144]) and the cingulate ([-14 16 43]) (Fig. 1). Most importantly, as for HF nonwords, no increased activation was detected in temporal brain regions, even when lowering the statistical threshold $(p<.001$, uncorrected). 


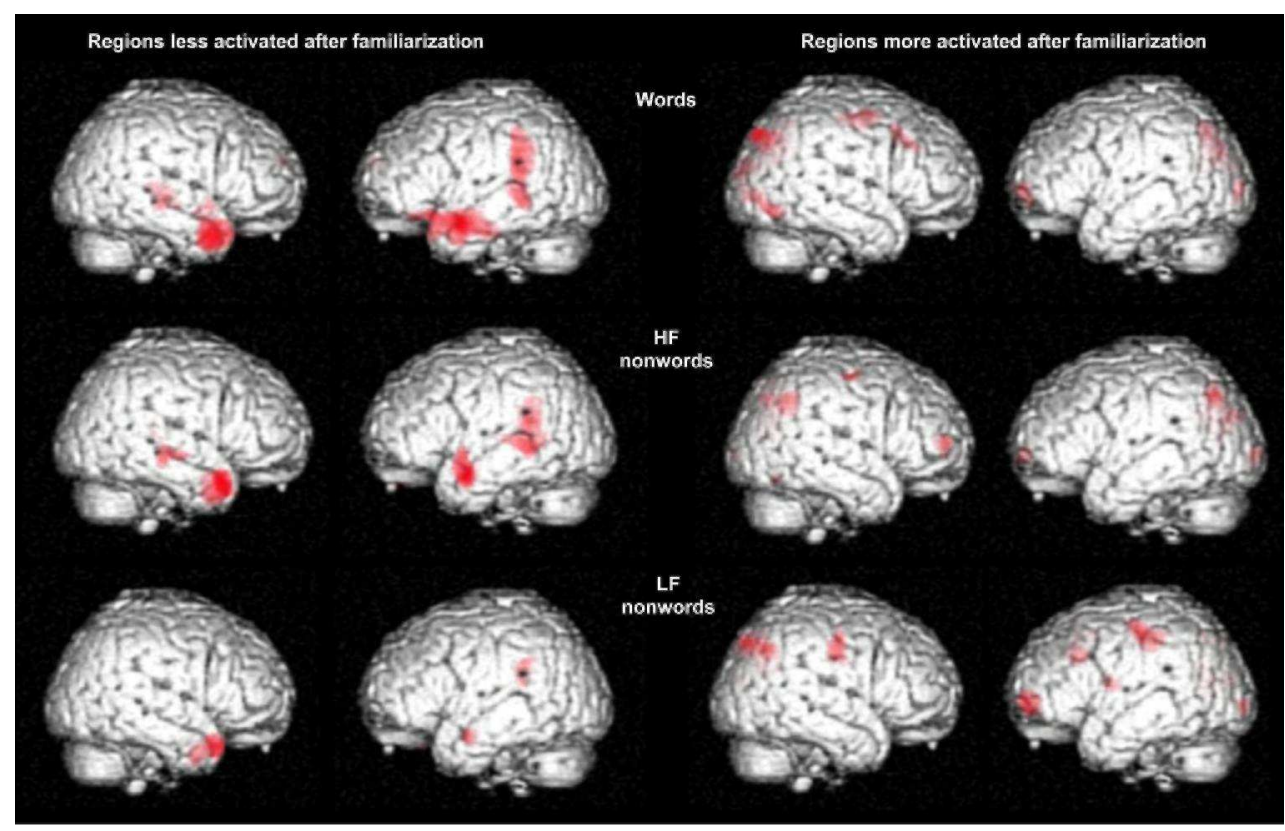

Fig. 1. Brain regions less or more activated after familiarization, as a function of stimulus condition ( $p$ $<.0001$ uncorrected, extend threshold: $k=50)$. Activation foci are rendered on a standard $3 D$ brain MRI.

\subsubsection{Interaction analyses}

A set of interaction analyses were performed in order to identify brain regions that are modulated differentially by familiarization, as a function of the linguistic status of the stimuli presented. Two areas in the superior temporal sulcus responded differentially to words, HF and LF nonwords: after familiarization, activation in the left and right posterior superior temporal sulcus was still more important for LF nonwords relative to words (contrast [famLF - LF] - [famW - W]) and relative to HF nonwords (contrast: [famLF - LF] - [famHF - HF]). This interaction does not mean that, in absolute terms, activity in these brain areas was greater after familiarization for LF nonwords, as in the subtraction analysis comparing brain activity for LF nonwords before and after familiarization, no increase in brain activity was observed in temporal regions after familiarization. Rather, the interaction means that the magnitude of decrease for LF nonwords after familiarization was less important than the decrease observed for HF nonwords and words. No other significant interactions were observed (see Table 5).

Table 5 Interaction analysis: Brain regions more or less activated for LF and HF nonwords after familiarization, compared to words

Brain area

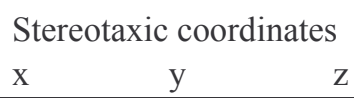

Z-value

$[\mathrm{famHF}-\mathrm{HF}]-[\mathrm{famW}-\mathrm{W}]$

No suprathreshold activation

$[$ famLF - LF] - [famW - W]

L Posterior STS (BA 22)

R Posterior STS (BA 22)

$\begin{array}{llll}-60 & -36 & 10 & 3.70 * \\ 69 & -23 & -2\end{array}$

$[\mathrm{famLF}-\mathrm{LF}]-[\mathrm{famHF}-\mathrm{HF}]$

69

$-23$

$-2$

$3.87^{*}$

L Posterior STS (BA 22)

R Posterior STS (BA 22)

$[$ famHF - HF] - [famLF - LF]

No suprathreshold activation

$[$ famW - W] - [famHF - HF $]$

No suprathreshold activation

$[$ famW - W] - [famLF - LF]

No suprathreshold activation

STS, superior temporal sulcus. ${ }^{*} \mathrm{p}<.05$, small volume corrections. 
Table 6 Correlation analysis: Brain regions in which familiarization-related change in activity correlates with the reduction of response latencies after familiarization

$\begin{array}{lllll}\text { Brain area } & \text { Stereotaxic coordinates } & \text { Z-value } \\ & \mathrm{x} & \mathrm{y} & \mathrm{Z} & \end{array}$

Words

No suprathreshold activation

HF nonwords

No suprathreshold activation

LF nonwords

L Posterior superior temporal (BA 22/40)

R Mid STS (BA 38)

R Posterior STS (BA 22)

$\begin{array}{llll} & -36 & 28 & 3.91_{-}^{*} \\ 55 & 9 & -16 & 4.52^{*} \\ 61 & -17 & 5 & 4.13^{*}\end{array}$

STS, superior temporal sulcus. ${ }^{*} p<.05$, small volume corrections.

\subsection{Correlation of familiarity-related brain activity and reaction times}

For word and HF nonwords, no significant correlations were observed between the size of the decrease of response latencies after familiarization and the contrast of brain activity before and after familiarization. However for LF nonwords, different activation foci were related to the decrease of response latencies after familiarization: the right posterior superior temporal sulcus, the left temporoparietal area, and the right temporal pole (see Table 6 and Fig. 2). Note that activation in the bilateral inferior parietal lobules and the right middle occipital gyrus was also observed at uncorrected p levels, as shown in Fig. 2. In all these regions, a larger decrease in response latencies was associated with smaller activation, relative to the mean activation level across all participants after familiarization (see Fig. 2). To sum up, for LF nonwords, those participants that showed the biggest familiarization effect at the level of response latencies were also those that showed the smallest activity in these regions after familiarization.

\section{Discussion}

The aim of this study was to investigate the neural processes that underlie phonological familiarization. Repetition of word, HF nonword, and LF nonword stimuli after a familiarization phase resulted in decreased activation in the left posterior superior temporal gyrus, the bilateral temporal pole, and bilateral middle temporal gyri. Interaction analyses showed that specifically for LF nonwords, the magnitude of this decrease of activation was significantly smaller in the bilateral posterior superior temporal sulcus, relative to both the word and HF nonword conditions. When regressing brain activity on familiarization-related decrease of reaction times for LF nonwords, a significant negative correlation was found between decrease of reaction times after familiarization and brain activity in the right anterior and posterior superior temporal sulcus and the left temporo-parietal area: a larger effect of familiarization at the behavioral level for LF nonwords was associated with smaller activation in these areas after familiarization. 

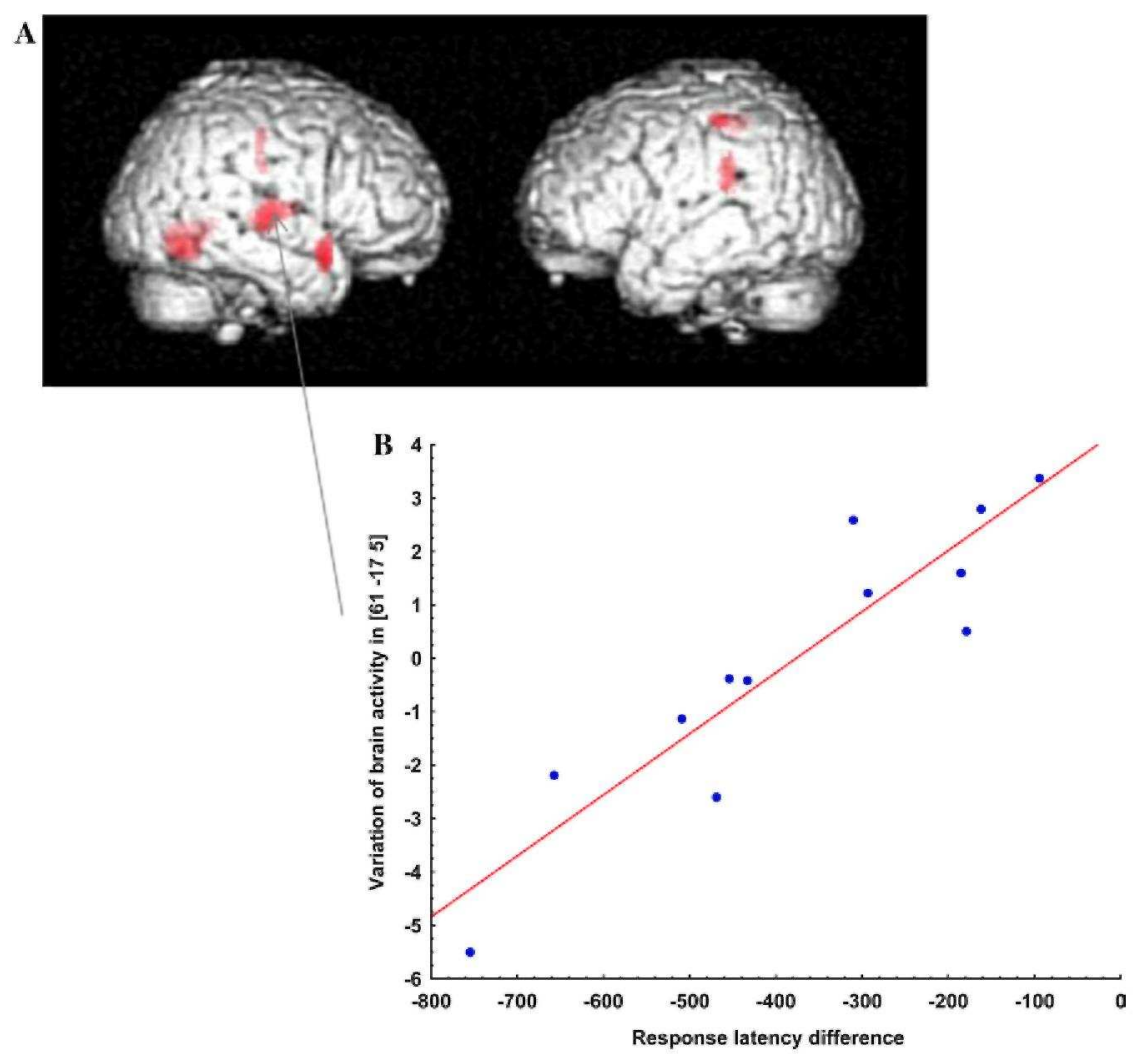

Fig. 2. Brain regions in which familiarization-related change in activity correlates with the reduction of response latencies after familiarization ( $p<.0001$, uncorrected; extend threshold: $k=50)(A)$. Activation foci are rendered on a standard $3 D$ brain MRI. A detailed illustration is provided for the correlation between response latencies and variation of activity in the right posterior superior temporal gyrus (coordinates: $61-175 ; R=.83)(B)$; similar linear relationships were also observed between response latencies and the other brain regions shown in (A).

Finally, increased activation was also observed after familiarization, but only in extra-temporal areas, including the right precuneus and posterior cingulate cortex.

As explained in Section 1, repetition priming is the experimental procedure that comes closest to the familiarization paradigm used in this study. The results, showing a global reduction of brain activity in language processing areas after familiarization to verbal stimuli is in accordance with previous studies on repetition priming which have also shown a reduction of brain response following the repeated presentation of the same verbal stimulus (Buckner et al., 2000; Haist et al., 2001; Mar-inkovic et al., 2003). However, in addition to previous work, our results show that this reduction is observed for both familiar and unfamiliar verbal information. In line with theoretical interpretations of repetition priming (e.g., Henson \& Rugg, 2003), the reduced activity observed in the posterior superior temporal region after familiarization might reflect faster, more focused and more efficient processing at the neural level, which, in this case, underlies the phonological identification of the target word and nonword stimuli; the posterior superior temporal gyrus and sulcus have indeed been shown to be involved in sub-word level phonological processing and analysis, as described in Section 1 (e.g., Binder et al., 2000; Jacquemot et al., 2003; Majerus et al., 2002; Mazoyer et al., 1993; Scott et al., 2000; Specht et al., 2003). Nevertheless, there might also be an alternative interpretation: the familiarization-related reduction of neural activity could also represent decreased reliance on phonological short-term memory storage during word and nonword repetition after familiarization. In fact, if after familiarization, the stimuli are identified and processed faster, there will be less need to keep them in an active short-term memory format for subsequent repetition. For the repetition of word and nonword stimuli without familiarization, the identification process is likely to be slower and subsequent processing stages at the lexico-semantic level (for words) and articulatory level (for words and especially non-words) will be activated less rapidly and smoothly, requiring the input information to be stored for a longer time in the form of a temporary short-term memory trace. Our interpretation is in agreement with a number of studies that have suggested that the posterior superior temporal sulcus and the temporo-parietal area play a role during the temporary maintenance of verbal information 
(Hickok \& Poeppel, 2000; Hughes et al., 2001; Mustovic et al., 2003; Wise et al., 2001). For example, in a recent study with three adult patients that had recovered from epileptic childhood aphasia (Landau-Kleffner syndrome), we showed that the patient who activated the posterior superior temporal gyrus showed good performance during repetition of four-word lists (compared to a singleword repetition control condition), while verbal short-term memory performance was deficient in the other two patients who showed lack of activity within the same area relative to healthy controls (Majerus et al., 2003).

Regarding the familiarization-related reduction of activity observed in the bilateral anterior and middle temporal lobes, for all three stimulus conditions, it most probably reflects more efficient ${ }^{2}$ processing at the lexical level, as these regions are consistently activated in tasks requiring lexico-semantic processing (e.g., Binder et al., 1996, 1999; Demonet et al., 1992; Majerus et al., 2002; Price et al., 1996; Scott et al., 2000). More precisely, in the case of words, lexico-semantic activation is likely to be broader before familiarization than after as, on the first presentation of a word, lexical competitors of the target word might also be activated, at least partially, in addition to the target lexical representation (see for example, Vitevitch \& Luce, 1998); this phenomenon is likely to disappear after repeated exposure to the same stimulus. For nonwords, lexical search processes might also be engaged during the initial processing stages before familiarization and are likely to be abandoned after familiarization. In addition to the general reduction of brain activity in phonological and lexico-semantic processing areas after familiarization, we also observed that familiarization-related activity interacted locally with stimulus familiarity: reduction of activation after familiarization was less important for LF nonwords relative to words and HF nonwords in the bilateral posterior superior temporal sulcus. This is in agreement with previous results obtained by Haist et al. (2001) in a repetition priming study with visually presented words and nonwords. As we have discussed relative to the posterior superior temporal area, a reduction of activity in this area after familiarization probably reflects more efficient sublexical phonological processing but possibly also reduced reliance on phonological short-term memory. Despite the fact that familiarity of LF nonwords increased after familiarization, LF nonwords are likely, nevertheless, to remain still less familiar than words and HF non-words whose familiarity also increased as a result of familiarization. Thus, relative to words and HF non-words, phonological identification after familiarization is likely to remain more demanding for LF nonwords and the need to maintain them in phonological short-term memory for subsequent repetition will also be greater. If this is true, then the reduction of neural activity after familiarization should be the strongest in those participants showing the largest behavioral familiarization effect. This is precisely what we observed in the correlation analysis, but only in the case of LF nonwords: the participants that presented the largest reduction of response latencies after familiarization also presented the strongest reduction of activity in a posterior area of the right posterior superior temporal sulcus (slightly anterior to the area observed in the interaction analysis) and in the left temporo-parietal area (slightly superior to the left posterior superior temporal sulcus activation observed in the interaction analysis). The right anterior superior temporal gyrus, another activation focus which also correlated with the reduction of response latencies after familiarization, is also in line with our interpretation as it is probably related to lexical search processes as explained above. These lexical search processes are indeed likely to be more engaged in those participants who get less easily familiarized with the LF nonwords.

A subsidiary question that arises at this level is why no significant correlations were observed between the behavioral familiarization effects and brain activity elicited after familiarization for words and HF nonwords. A possible explanation might be related to the fact that words, HF nonwords, and LF nonwords already differ in their initial degree of stimulus familiarity. The phonological familiarization processes associated with repeated exposure to words and HF nonwords are less likely to reveal a large inter-individual variability in phonological familiarization abilities, as these stimuli are already relatively familiar right from the beginning, and thus they are likely to put relatively few demands on phonological familiarization processes. The inter-individual variability observed in familiarizationrelated response latency differences for these stimuli may be caused by some independent factors such as distractibil-ity rather than inter-individual differences in phonological familiarization abilities. These differences are more likely to be revealed by familiarization to LF nonwords, as these items contain phoneme combinations that have a very low phonotactic frequency, and are thus initially much harder to process.

Finally, we might wonder whether some episodic long-term memory retrieval processes could also have become activated after familiarization. It is indeed likely that after familiarization, the participants began to explicitly recognize the items which had been presented six times during the familiarization phase. Also, when hearing the first few phonemes of a familiarized stimulus, an episodic memory trace of the entire stimulus could have been retrieved, in a similar way to what occurs in cued recall tasks. Partial support for this interpretation is provided by the results from a debriefing session that followed 
the imaging experiment. We asked the participants to recall any word or nonword they could remember. The participants managed indeed to recall on average seven words and one nonword from the familiarization lists, but virtually no word ( 0.33 word) and no nonword (at all) from the nonfamiliarization lists. These results suggest that at least some of the stimuli presented during familiarization had been encoded in episodic memory. The brain regions that showed increased activation after familiarization are compatible with this suggestion. We indeed observed increased activation in the precuneus and posterior cingu-late, as well, at uncorrected significance levels, in superior and middle frontal gyri, inferior parietal lobules, and inferior occipital gyri. This network is indeed similar to the network typically involved in episodic memory tasks (see for example, Cabeza \& Nyberg, 2000, for a review).

In summary, our data indicate the existence of two different phenomena as a result of familiarization to phonological information: (1) a general reduction of brain activity in those posterior superior and anterior middle temporal areas that subtend the initial sublexical and lexical phonological processing of the stimuli; (2) in the bilateral posterior superior temporal gyrus, however, this reduction of activity interacts with initial stimulus familiarity: more unfamiliar the verbal item initially is, the less important the reduction of brain activity after familiarization will be. This is possibly related to phonological identification and/or short-term memory storage processes that will remain more active for those items for which it is harder to get familiarized.

\section{Acknowledgments}

This work was supported by the Belgian National Fund for Scientific Research (FNRS), the 'Fondation Medicale Reine Elisabeth,' and the Interuniversity Attraction Poles Program P5/04, Belgian Science Policy.

Steve Majerus is a Postdoctoral Researcher and Fabi-enne Collette and Steven Laureys are Research Associates, each of them for the Belgian National Fund for Scientific Research (FNRS). We gratefully acknowledge the statistical assistance of Philippe Peigneux and the comments of two anonymous reviewers.

\section{Notes}

1 We only used words matched to HF nonwords at the level of diphone frequency. Although it would also have been possible to include words with less frequent diphone frequency patterns, it would have been very difficult to match them to the (very low) diphone frequencies of the LF nonwords used, unless raising again the diphone frequencies of the LF nonwords. In that case, the difference in diphone frequency between HF and LF nonwords would have become much smaller, reducing the likelihood of observing any difference at the level of behavioral and neural processing between these two types of stimuli. Furthermore, including a fourth stimulus condition would have needed to add four scans, raising the total scan number to 16 , which is impracticable given the time constraints imposed by PET methodology.

${ }^{2}$ We have interpreted the familiarization-related decrease of brain activation as reflecting more efficient processing at the neural level in different regions underlying phonological and lexico-semantic processing. A stronger hypothesis would be to assume that processing in the whole language network is more efficient, and that transmission of information between the different brain regions would also become more coordinated. We reanalyzed our data using effective connectivity analysis (Büchel, Coull, \& Friston, 1999) in order to determine whether the different regions in the superior and middle temporal lobes that responded to familiarization were more strongly correlated after than before familiarization. However, the results of this reanalysis did not support this stronger hypothesis: no difference in strength of correlations between left posterior superior temporal and bilateral middle temporal lobes was observed after familiarization.

\section{References}

Awh, E., Jonides, J., Smith, E. E., Schumacher, E. H., Koeppe, R. A., \& Katz, S. (1996). Dissociation of storage and rehearsal in verbal working memory: Evidence from positron emission tomography. Psychological Science, 7, 25-31.

Binder, J. R., Frost, J. A., Hammeke, T. A., Bellgowan, P. S. F., Rao, S. M., \& Cox, R. W. (1999). Conceptual processing during the conscious resting state: a functional MRI study. Journal of Cognitive Neuroscience, 11, 80-93. 
Binder, J. R., Frost, J. A., Hammeke, T. A., Bellgowan, P. S. F., Springer, J. A., Kaufman, J. N., et al. (2000). Human temporal lobe activation by speech and nonspeech sounds. Cerebral Cortex, 10, 512-528.

Binder, J. R., Frost, J. A., Hammeke, T. A., Rao, S. M., \& Cox, R. W. (1996). Function of the left planum temporale in auditory and linguistic processing. Brain, 119, 1239-1247.

Binder, J. R., \& Price, C. (2001). Functional neuroimaging of language. In R. Cabeza \& A. Kingstone (Eds.), Handbook of functional neuroimaging of cognition (pp. 187-252). Cambridge, MA: MIT Press.

Booth, J. R., Burman, D. D., Van Santen, F. W., Harasaki, Y., Gitelman, D. R., Parrish, T. B., et al. (2001). The development of specialized brain systems in reading and oral-language. Child Neuropsychology, 7, 119-141.

Breitenstein, C., \& Knecht, S. (2002). Development and validation of a language learning model for behavioral and functionalimaging studies. Journal of Neuroscience Methods, 114, 173-179.

Büchel, C., Coull, J. T., \& Friston, K. J. (1999). The predictive value of changes in effective connectivity for human learning. Science, 283, 1341-1538.

Buckner, R. L., Koutstaal, W., Schacter, D. L., \& Rosen, B. R. (2000). Functional MRI evidence for a role of frontal and inferior temporal cortex in amodal components of priming. Brain, 123, 620-640.

Burton, M. W., Small, S. L., \& Blumstein, S. E. (2000). The role of segmentation in phonological processing: An fMRI investigation. Journal of Cognitive Neuroscience, 12, 679-690.

Cabeza, R., \& Nyberg, L. (2000). Imaging cognition II: an empirical review of 275 PET and fMRI studies. Journal of Cognitive Neuroscience, 12, 1-47.

Clark, D., \& Wagner, A. D. (2003). Assembling and encoding word representations: fMRI subsequent memory effects implicate a role for phonological control. Neuropsychologia, 41, 304-317.

Cornelissen, K., Laine, M., Tarkiainen, A., Jarvensivu, T., Martin, N., \& Salmelin, R. (2003). Adult brain plasticity elicited by anomia treatment. Journal of Cognitive Neuroscience, 15, 444-461.

Davachi, L., Maril, A., \& Wagner, A. D. (2001). When keeping in mind supports later bringing to mind: Neural markers of phonological rehearsal predict subsequent remembering. Journal of Cognitive Neuroscience, 13, 1059-1070.

Dehaene-Lambertz, G., Dehaene, S., \& Hertz-Pannier, L. (2002). Functional neuroimaging of speech perception in infants. Science, 298, 2013-2015.

Demonet, J. F., Chollet, F., Ramsay, S., Cardebat, D., Nespoulous, J. L., Wise, R. J. S., et al. (1992). The anatomy of phonological and semantic processing in normal subjects. Brain, 115, 1753-1768.

Demonet, J. F., Price, C., Wise, R. J. S., \& Frackowiak, R. S. J. (1994). Differential activation of right and left posterior sylvian regions by semantic and phonological tasks: A positron emission tomography study in normal human subjects. Neuroscience Letters, 182, 25-28.

Friston, K. J., Ashburner, J., Frith, C. D., Poline, J. B., Heather, J. D., \& Frackowiak, R. S. J. (1995a). Spatial realignment and normalization of images. Human Brain Mapping, 2, 165-189.

Friston, K. J., Holmes, A. P., Worsley, K. J., Poline, J. B., Frith, C. D., \& Frackowiak, R. S. J. (1995b). Statistical parametric maps in functional imaging: A general linear approach. Human Brain Mapping, 2, 189-210.

Haist, F., Song, A. W., Wild, K., Faber, T. L., Popp, C. A., \& Morris, R. D. (2001). Linking sight and sound: fMRI evidence of primary auditory cortex activation during visual word recognition. Brain and Language, 76, 340-350.

Henson, R. N., \& Rugg, M. D. (2003). Neural response suppression, haemodynamic repetition effects, and behavioural priming. Neuro-psychologia, 41, 263-270.

Hickok, G., Erhard, P., Kassubek, J., Helms-Tillery, A. K., Naeve-Velguth, S., Strupp, J. P., Strick, P. L., \& Ugurbil, K. (2000). A functional magnetic resonance imaging study of the role of left posterior superior temporal gyrus in speech production: implications for the explanation of conduction aphasia. Neuroscience Letters, 287, 156-160.

Hickok, G., \& Poeppel, D. (2000). Towards a functional neuroanat-omy of speech perception. Trends in Cognitive Sciences, 4, $131-138$.

Holland, S. K., Plante, E., Weber Byars, A., Strawsburg, R. H., Schmithorst, V. J., \& Ball, W. S. (2001). Normal fMRI brain activation patterns in children performing a verb generation task. NeuroImage, 14, 837-843.

Holmes, A., \& Friston, K. (1998). Generalisability, random effects and population inference. NeuroImage, 7, 754-754.

Howard, D., Patterson, K., Wise, R. J. S., Brown, W. D., Friston, K. J., Weiller, C., et al. (1992). The cortical localization of the lexicons. Brain, 115, 1769-1782.

Hughes, H. C., Darcey, T. M., Barkan, H. I., Williamson, P. D., Roberts, D. W., \& Aslin, C. H. (2001). Responses of human 
auditory association cortex to the omission of an expected acoustic event. NeuroImage, 13, 1073-1089.

Jacquemot, C., Pallier, C., Le Bihan, D., Dehaene, S., \& Dupoux, E. (2003). Phonological grammar shapes the auditory cortex: A functional magnetic resonance imaging study. Journal of Neuro-science, 23, 9541-9546.

Ja"ncke, L., Wu“stenberg, T., Scheich, H., \& Heinze, H.-J. (2002). Phonetic perception and the temporal cortex. NeuroImage, 15, $733-746$.

Joanisse, M. F., \& Gati, J. S. (2003). Overlapping neural regions for processing rapid temporal cues in speech and nonspeech signals. NeuroImage, 19, 64-79.

Jonides, J., Schumacher, E. H., Smith, E. E., Koeppe, R. A., Awh, E., Reuter-Lorenz, P. A., et al. (1998). The role of parietal cortex in verbal working memory. Journal of Neuroscience, 18, 5026-5034.

Jueptner, M., \& Weiller, C. (1995). Review: Does measurement of regional cerebral blood flow reflect synaptic activity? Implications for PET and fMRI. NeuroImage, 2, 148-156.

Leger, A., Demonet, J.-F., Ruff, S., Aithamon, B., Touyeras, B., Puel, M., et al. (2002). Neural substrates of spoken language rehabilitation in an aphasic patient: An fMRI study. NeuroImage, 17, 174-183.

Majerus, S., Collette, F., Van der Linden, M., Peigneux, P., Laureys, S., Delfiore, G., et al. (2002). A PET investigation of lexicality and phonotactic frequency in oral language processing. Cognitive Neuropsychology, 19, 343-360.

Majerus, S., Laureys, S., Collette, F., Del Fiore, G., Degueldre, C., Luxen, A., et al. (2003). Phonological short-term memory networks following recovery from Landau and Kleffner syndrome. Human Brain Mapping, 19, 133-144.

Majerus, S., Van derLinden, M., Mulder, L., Meulemans, T., \& Peters, F. (2004). Verbal short-term memory reflects the sublexical organization of the phonological language network. Evidence

from an incidental phonotactic learning paradigm. Journal of Memory and Language, 51, 297-306.

Marinkovic, K., Dhond, R. P., Dale, A. M., Glessner, M., Carr, V., \& Halgren, E. (2003). Spatiotemporal dynamics of modalityspecific and supramodal word processing. Neuron, 38, 487-497.

Mazoyer, B., Tzourio, N., Frak, V., Syrota, A., Murayama, N., Levrier, O., et al. (1993). The cortical representation of speech. Journal of Cognitive Neuroscience, 5, 467-479.

Mummery, C. J., Ashburner, J., Scott, S. K., \& Wise, R. J. S. (1999).Functional neuroimaging of speech perception in six normal and two aphasic subjects. Journal of the Acoustical Society of America, 106, 449-457.

Musso, M., Weiller, C, Kiebel, S., Mu“ller, S. P., Bülau, P., \& Rijntjes, M. (2000). Training-induced brain plasticity in aphasia. Brain, 122, 1781-1790.

Mustovic, H., Scheffler, K., Di Salle, F., Esposito, F., Neuhoff, J. G., Hennig, J., et al. (2003). Temporal integration of sequential auditory events: silent period in sound pattern activates human planum temporale. NeuroImage, 20, 429-434.

Paulesu, E., Frith, C. D., \& Frackowiak, R. S. J. (1993). The neural correlates of the verbal component of working memory. Nature, 362, 342-345.

Perani, D., Dehaene, S., Grassi, F., Cohen, L., Cappa, S. F., Dupoux, E., et al. (1996). Brain processing of native and foreign languages. NeuroReport, 7, 2439-2444.

Petersen, S. E., Fox, P. T., Posner, M. I., Mintun, M., \& Raichle, M. E. (1988). Positron emission tomographic studies of the cortical anatomy of single-word processing. Nature, 331, 585-589.

Petersen, S. E., Fox, P. T., Posner, M. I., Mintun, M., \& Raichle, M. E. (1989). Positron emission tomographic studies of the processing of single words. Journal of Cognitive Neuroscience, 1, 153-170.

Price, C. J., Wise, R. J. S., Warburton, E. A., Moore, C. J., Howard, D., Patterson, K., et al. (1996). Hearing and saying? The functional neuro-anatomy of auditory word processing. Brain, 119, 919-931.

Saffran, J. R., Aslin, R. N., \& Newport, E. L. (1996a). Statistical learning by 8-month-old infants. Science, 274, $1926-1928$.

Saffran, J. R., Newport, E. L., \& Aslin, R. N. (1996b). Word segmentation: The role of distributional cues. Journal of Memory and Language, 35, 606-621.

Saffran, J. R., Newport, E. L., Aslin, R. N., \& Tunick, R. A. (1997). Incidental language learning: Listening (and learning) out of the corner of your ear. Psychological Science, 8, 101-105.

Scott, S. K., Catrin Blank, C, Rosen, S., \& Wise, R. J. S. (2000). Identification of a pathway for intelligible speech in the left temporal lobe. Brain, 123, 2400-2406.

Smith, E. E., \& Jonides, J. (1998). Neuroimaging analyses of human working memory. Proceedings of the National Academy of Sciences of the Unites States of America, 95, 12061-12068. 
Published in: Brain and language (2005), vol. 92, iss. 3, pp. 320-331

Status: Postprint (Author's version)

Specht, K., Holtel, C, Zahn, R., Herzog, H., Krause, B. J., Motthagy, F. M., et al. (2003). Lexical decision of nonwords and pseudo-words in humans: A positron emission tomography study. Neuro-science Letters, 345, 177-181.

Talairach, J., \& Tournoux, P. (1988). Co-planar stereotaxic atlas of the human brain. Stuttgart: Thieme Medical Publishers. Thompson, C. K. (2000). Neuroplasticity: Evidence from aphasia. Journal of Communication Disorders, 33, $357-366$.

Tubach, J. L., \& Boe, L. J. (1990). Un corpus de transcription phonetique. France: Telecom. Vitevitch, M. S., \& Luce, P. A. (1998). When words compete: Levels of processing in perception of spoken words. Psychological Science, 9, 325-329.

Wise, R. J. S., Scott, S. K., Blank, S. C, Mummery, C. J., Murphy, K., \& Warburton, E. A. (2001). Separate neural subsystems within 'Wernicke's area'. Brain, 124, 83-95. 